\title{
Application of the Package SIESTA to Linear Models of a Molecular Chromium-Based Ring
}

\author{
T. Ślusarski ${ }^{a}$, B. Brzostowski ${ }^{b, *}$, D. TomeckA ${ }^{a}$ And G. KAMieniarz ${ }^{a}$ \\ ${ }^{a}$ Faculty of Physics, A. Mickiewicz University, Umultowska 85, 61-614 Poznań, Poland \\ ${ }^{b}$ Institute of Physics, University of Zielona Góra, Prof. Z. Szafrana 4a, 65-516 Zielona Góra, Poland \\ We investigate for the first time the electronic and magnetic properties of the linear models of $\mathrm{Cr}_{8} \mathrm{~F}_{8}\left(\mathrm{Piv}_{16}\right.$ \\ molecular ring using the SIESTA package. In the first step the proper values of the SIESTA parameters and \\ the optimal basis set needed for the convergence of the total energy are established. Next the estimates of the \\ magnetic coupling $J$ confirming the previous density functional theory calculations are presented.
}

PACS numbers: 75.50.Xx, 71.15.Mb, 73.22.-f, 75.30.Et

\section{Introduction}

Molecular chromium-based wheels are fascinating magnetic materials. These spin rings constitute a rich subgroup among magnetic molecules. These regular structures of various sizes which can comprise a large variety of paramagnetic ions are ideal investigation objects for better understanding electronic and magnetic properties of whole class of magnetic molecules, especially containing antiferromagnetically coupled spin systems.

In this paper we study electronic and magnetic properties of $\mathrm{Cr}_{8} \mathrm{~F}_{8}(\mathrm{Piv})_{16}$ molecular ring (in short $\mathrm{Cr}_{8}$ ) using SIESTA package $[1,2]$ and the linear models $[3,4]$. SIESTA uses linear combination of numerical localized atomic-orbital basis sets for the description of valence electrons and norm-conserving non-local pseudopotentials for the atomic core. Exchange and correlation effects are accounted for by the generalized gradient local approximation (GGA).

The motivation for this work was to examine whether the simple linear models, which are computationally much more convenient with respect to the original $\mathrm{Cr}_{8}$ molecule [3], can give reliable results for $\mathrm{Cr}_{8}$. Another goal is to check if calculations based on the SIESTA code give reliable results consistent with those obtained by other codes based on density functional theory (DFT) and with experiment [3-5]. As far as we know this is the first attempt to use the SIESTA code to analysis of the chromium-based molecular magnets, although some applications to other systems have been undertaken (e.g. [6]).

The linear models for $\mathrm{Cr}_{8}$ are proposed in [3] and are determined by the corresponding repetition units which are different segments of the $\mathrm{Cr}_{8}$ ring. Following [3], the models are denoted as chain, zigzag and snake.

\section{Results and discussion}

We carry out the SIESTA calculations using the same geometrical structures as those in [3] and exploit exchange-correlation potential proposed by Padrew,

\footnotetext{
* corresponding author; e-mail: brzost@proton.if.uz.zgora.pl
}

Burke and Ernzerhof (PBE) [7] as well as BLYP i.e correction of Becke [8] and the correlation function of Lee, Yang and Parr [9]. We consider only ferromagnetic (FM) and antiferromagnetic (AFM) spin configurations of the $\mathrm{Cr}$ (III) ions in all linear models. By comparing the total energies, we obtain that the AFM configuration corresponds to the ground state. We estimate the magnetic coupling $J$ from the difference between the energies of the ground state and the excited FM configuration [3]. Thus the difference $\Delta E=E_{\mathrm{FM}}-E_{\mathrm{AFM}}$ is the crucial quantity and we give the evidence that its estimate within SIESTA is reliable.

As shown by a number of experiments and model calculations [3, 10-13], the exchange coupling between the $\mathrm{Cr}$ (III) ions is of the order of a few meV so that there is a need to reach the accuracy of the $\Delta E$ estimate of the order of a few tenths of meV.

In Table I the total energy difference $\Delta E=E_{\mathrm{FM}}-$ $E_{\mathrm{AFM}}$ and the corresponding values of exchange interaction parameter $J$ for different basis sets are presented. The basis sets are denoted as follows: SZP stands for the single- $\zeta$ polarized, DZP - for double- $\zeta$ polarized, TZP - triple- $\zeta$ polarized, TZDP - triple- $\zeta$ double polarized and TZTP - triple- $\zeta$ triple polarized. As expected, we reach the required accuracy and stability of $\Delta E$ for the more extended basis sets [2].

TABLE I

Exchange interaction parameter $J$ and total energy difference between the FM and AFM configurations for chain model with different basis sets. Density matrix tolerance and mesh cut-off set to $10^{-6}$ and $500 \mathrm{Ry}$, respectively.

\begin{tabular}{c|c|c|c|c|c}
\hline \hline & SZP & DZP & TZP & TZDP & TZTP \\
\hline$J[\mathrm{meV}]$ & 8.312 & 6.518 & 6.241 & 6.251 & 6.224 \\
$E_{\mathrm{FM}}-E_{\mathrm{AFM}}[\mathrm{meV}]$ & 74.81 & 58.66 & 56.17 & 56.26 & 56.01
\end{tabular}

In Table II the values of exchange interaction parameter $J$ for the chain model with DZP basis set for different mesh cut-off (MCO), expressed in Ry, and density matrix tolerance (DMT) parameters are reported. Default 
values of these parameters in SIESTA are $100 \mathrm{Ry}$ and $10^{-4}$, respectively, but $\mathrm{MCO} \geq 200 \mathrm{Ry}$ is generally used (e.g. [6]), while DMT parameter is chosen depending on the required accuracy. It is clearly shown for more exacting values the excellent stability of the estimates for $\mathrm{MCO} \geq 300$ and DMT $<10^{-4}$. These estimates agree very well with those found by the DFT calculations in $[3,5]$ using the WIEN2k package.

TABLE II

Exchange interaction parameter $J$ with different mesh cut-off and DMT parameters.

\begin{tabular}{c|c|c|c|c}
\hline \hline \multirow{2}{*}{ Mesh cut-off [Ry] } & \multicolumn{4}{|c}{ DM tolerance } \\
\cline { 2 - 5 } & $10^{-4}$ & $10^{-5}$ & $10^{-6}$ & $10^{-7}$ \\
\hline 300 & 6.832 & 6.526 & 6.500 & 6.502 \\
500 & 6.537 & 6.529 & 6.518 & 6.517 \\
700 & 6.514 & 6.523 & 6.521 & 6.511
\end{tabular}

It is interesting to calculate the local magnetic moments localized on the $\mathrm{Cr}$ (III) ions. The experiments and model calculations $[3,10-13]$ give the value $3 \mu_{\mathrm{B}}$ whereas the DFT calculations based on the integration inside the muffin-tin sphere $[3,5]$ lead to the quantitative estimate $2.7 \mu_{\mathrm{B}}$. Our results are calculated both by integration within the muffin-tin sphere with a given Bohr radius 2.38 and by the Mulliken analysis, and are presented in Table III. The muffin-tin values agree with those found in $[3,5]$, whereas the Mulliken analysis leads to better estimates. In both cases some localization of the spin density on the oxygen and fluorine sites is detected.

TABLE III

HOMO-LUMO majority and minority gaps and Cr magnetic moments for the linear models of $\mathrm{Cr}_{8}$ molecule in the AFM and FM configurations (for DZP basis set with $\mathrm{MCO}=500 \mathrm{Ry}$ and $\left.\mathrm{DMT}=10^{-6}\right)$.

\begin{tabular}{c|c|c|c|c|c|c}
\hline \hline & \multicolumn{2}{|c|}{ Chain } & \multicolumn{2}{c|}{ Zigzag } & \multicolumn{2}{c}{ Snake } \\
\cline { 2 - 7 } & AFM & FM & AFM & FM & AFM & FM \\
\hline$E_{\mathrm{H}-\mathrm{L}}^{\mathrm{maj}}[\mathrm{eV}]$ & 2.21 & 1.99 & 2.31 & 2.15 & 2.35 & 2.12 \\
$E_{\mathrm{H}-\mathrm{L}}^{\mathrm{min}}[\mathrm{eV}]$ & 2.23 & 3.73 & 2.34 & 3.26 & 2.27 & 3.31 \\
$m_{\mathrm{Cr}}^{\mathrm{m}-\mathrm{t}}\left[\mu_{\mathrm{B}}\right]$ & -2.704 & +2.751 & -2.697 & +2.767 & -2.712 & +2.767 \\
& +2.699 & +2.757 & +2.712 & +2.746 & +2.696 & +2.747 \\
$m_{\mathrm{Cr}}^{\mathrm{Mul}}\left[\mu_{\mathrm{B}}\right]$ & -2.887 & +2.923 & -2.877 & +2.944 & -2.895 & +2.944 \\
& +2.879 & +2.931 & +2.896 & +2.918 & +2.876 & +2.919
\end{tabular}

The magnetic moments are supplemented by the results for the highest occupied molecular orbital (HOMO) and the lowest unoccupied molecular orbital (LUMO) gaps for both spin channels. In AFM state HOMOLUMO gaps $E_{\mathrm{H}-\mathrm{L}}^{\mathrm{maj}}$ and $E_{\mathrm{H}-\mathrm{L}}^{\mathrm{min}}$ for majority and minority spin channel are almost the same, while for FM configuration we observe two clear spin channels with different HOMO-LUMO gaps. Our results are in good agreement with other theoretical investigations $[3,5]$.

\section{Conclusions}

We have carried out the systematic SIESTA-based DFT calculations for the linear models of the $\mathrm{Cr}_{8}$ molecule. We have shown that HOMO-LUMO gaps, magnetic moments and exchange couplings are in quantitative agreement with other DFT theoretical investigations. Our analysis has confirmed that the linear models describe very well the electronic and magnetic properties of the molecule $\mathrm{Cr}_{8}$.

\section{Acknowledgments}

The numerical computations were performed in the PSNC Supercomputing Center in Poznań. This work has been supported by the grant No. N N202 230137 from the Ministry of Science and Higher Education (Poland).

\section{References}

[1] P. Ordejón, E. Artacho, J.M. Soler, Phys. Rev. B (Rapid Commun.) 53, R10441 (1996).

[2] J.M. Soler, E. Artacho, J.D. Gale, A. García, J. Junquera, P. Ordejón, D. Sánchez-Portal, J. Phys., Condens. Matter 14, 2745 (2002).

[3] D.M. Tomecka, V. Bellini, F. Troiani, F. Manghi, G. Kamieniarz, M. Affronte, Phys. Rev. B 77, 224401 (2008).

[4] V. Corradini, F. Moro, R. Biagi, V. De U. Renzi, del Pennino, V. Bellini, S. Carretta, P. Santini, V.A. Milway, G. Timco, R.E.P. Winpenny, M. Affronte, Phys. Rev. B 79, 144419 (2009).

[5] V. Bellini, A. Olivieri, F. Manghi, Phys. Rev. B 73, 184431 (2006).

[6] J. Cano, R. Costa, S. Alvarez, E. Ruiz, J. Chem. Theory Comput. 3, 782 (2007).

[7] J. P. Perdew, K. Burke, M. Ernzerhof, Phys. Rev. Lett. 77, 3865 (1996).

[8] A.D. Becke, Phys. Rev. A 38, 3098 (1988).

[9] C. Lee, W. Yang, R.G. Parr, Phys. Rev. B 37, 785 (1988).

[10] S. Carretta, J. van Slageren, T. Guidi, E. Liviotti, C. Mondelli, D. Rovai, A. Cornia, A.L. Dearden, F. Carsughi, M. Affronte, C.D. Frost, R.E.P. Winpenny, D. Gatteschi, G. Amoretti, R. Caciuffo, Phys. Rev. B 67, 094405 (2003).

[11] G. Kamieniarz, P. Kozłowski, G. Musiał, W. Florek, M. Antkowiak, M. Haglauer, A. Caramico, D'Auria, F. Esposito, Inorg. Chim. Acta 361, 3690 (2008).

[12] P. Kozłowski, G. Kamieniarz, M. Antkowiak, F. Tuna, G.A. Timco, R.E.P. Winpenny, Polyhedron 28, 1852 (2009).

[13] S. Piligkos, H. Weihe, E. Bill, F. Neese, H. El Mkami, G.M. Smith, D. Collison, G. Rajaraman, G.A. Timco, R.E.P. Winpenny, E.J.L. McInnes, Chem. Eur. J. 15, 3152 (2009). 